\title{
Minimal entropic kinetic models for simulating hydrodynamics
}

\author{
Santosh Ansumali, Iliya V. Karlin, If and Hans Christian Öttinger \\ ETH-Zürich, Department of Materials, Institute of Polymers \\ ETH-Zentrum, Sonneggstr. 3, ML J 19, CH-8092 Zürich, Switzerland
}

\begin{abstract}
We derive minimal discrete models of the Boltzmann equation consistent with equilibrium thermodynamics, and which recover correct hydrodynamics in arbitrary dimensions. A simple analytical procedure of constructing the equilibrium for the nonisothermal hydrodynamics is established. A new discrete velocity model is proposed for the simulation of the Navier-Stokes-Fourier equation and is tested in the set up of Taylor vortex flow. For the lattice Boltzmann method of isothermal hydrodynamics, the explicit analytical form of the equilibrium distribution is presented.
\end{abstract}

PACS numbers: 05.70.Ln, $47.11 .+\mathrm{j}$ 
Minimal kinetic models, and primarily the lattice Boltzmann method (hereafter LBM), have recently met with significant success in simulations of complex isothermal hydrodynamic phenomena. A few examples of the successful application are the simulation of fluidparticle suspensions, turbulent flows, spinodal decomposition [四, 2, 3]. The first large-scale simulations of 3D spinodal decomposition in inertial regime [2] and simulation of Brownian short-time regime [ [i] are a few successes achieved in the field of computational fluid mechanics through use of these approaches. The ability to handle a very complicated geometry in a very simple manner has allowed these method to emerge as an alternative to purely continuum approaches, even for solving isothermal Navier-Stokes equation [3]. In these methods, hydrodynamic equations (for example, the isothermal Navier-Stokes and the nonisothermal Navier-Stokes-Fourier equations in our terminology) are not addressed by a direct discretization procedure. Instead a simplified kinetic equation is introduced in such a way that hydrodynamic equations are obtained as its large-scale long-time limit. Two central issues in the construction of such models are the choice of discrete velocities, as few as possible, and the construction of the local equilibria such that the desired hydrodynamic equations are reproduced as closely as possible by the kinetic model.

Unlike the isothermal case, the kinetic modeling of the nonisothermal hydrodynamics is a hitherto unsolved problem [1, 5]. The kinetic models with proper thermodynamics are especially needed for the simulation of chemically reactive flows and the multiphase flows and near continuum flows in microdevices, which are difficult to simulate using a purely continuum models. However, the nonisothermal model is not established even in the case of the Navier-Stokes-Fourier dynamics of a single fluid. Apart from the conserved moments of distribution function, the mass, the momentum and the energy, also non-conserved moments, the stress tensor, the heat flux and fourth moments need to be in a specific form to recover the Navier-Stokes-Fourier equations. This was previously accomplished by assuming a predefined simple functional form, typically a polynomial, for the equilibrium population [1]. In such a setting, the construction is neither unique in the choice of the discrete velocity set, nor in the choice of the function. Moreover, these schemes permit populations to attain negative values and thus make the simulation scheme unstable [5, 6]. The way to resolve the problem of non-positive form of the population is to define the equilibrium population as a minimum of a convex function, known as $H$ function, under the constraint of local conservation laws. Recently, the advantage of such an approach was shown in the context of two dimensional 
isothermal hydrodynamics [7, 8, 9, 10]. In order to avoid an explicit calculation of the local equilibria, these studies took an alternative root of using computationally intensive kinetic equations.

Apart from the stability issue, another well known problem associated with the current discrete velocity models is non-adherence to the equation of the state [11]. In these models, the local equilibrium entropy does not satisfy the usual definition of the temperature as a function of the entropy and the energy known from the elementary thermodynamics [11].

In this letter, we construct kinetic models, which are free from all the problems discussed above, in arbitrary dimensions. The proper choice of the set of the discrete velocities and the $H$ function and the explicit expression for the equilibrium are the main result of the present work. These models retain the simplicity and computational efficiency of the standard lattice Boltzmann model. Further, for the isothermal lattice Boltzmann method an explicit equilibria with correct $H$ theorem is derived.

Before constructing such model, we briefly explain the basic setup of the discrete velocity models. In these methods, the kinetic equation is written for the populations $f_{i}(\mathbf{x}, t)$ of the discrete velocities $\mathbf{c}_{i}, i=1, \ldots, b$, defined at position $\mathbf{x}$ and time $t$. Hydrodynamic fields are first few moments of populations, namely $\rho=\sum_{i=1}^{b} f_{i}$ (density), $\rho u_{\alpha}=\sum_{i=1}^{b} f_{i} \mathbf{c}_{i \alpha}$ (momentum density, $\alpha=1, \ldots, D$, where $D$ is the spatial dimension), and $\rho D T+\rho u^{2}=$ $\sum_{i=1}^{b} f_{i} \mathbf{c}_{i}^{2}$ (energy density). In the case of isothermal hydrodynamics, the hydrodynamic fields include $\rho$ and $\rho u_{\alpha}$, whereas in the nonisothermal case the energy density is also included as independent field. Typical model kinetic equation reads,

$$
\partial_{t} f_{i}+c_{i \alpha} \partial_{\alpha} f_{i}=-\tau^{-1}\left(f_{i}-f_{i}^{\mathrm{eq}}\right),
$$

where the model collision integral on the right hand side is assumed in the Bhatnagar-GrossKrook (BGK) form [12], with $\tau$ as the relaxation time. The collision integral must respect local conservation laws which imply (in the nonisothermal case),

$$
\sum_{i=1}^{b} f_{i}^{\mathrm{eq}}\left\{1, c_{i \alpha}, c_{i}^{2}\right\}=\left\{\rho, \rho u_{\alpha}, \rho D T+\rho u^{2}\right\}
$$

In the isothermal case the energy constraint on the local equilibrium is excluded from this list. Besides Eq. (2), the local equilibrium must respect several other conditions for the nonconserved fields (examples will be given below). These latter conditions are found on the basis of the Chapman-Enskog analysis of the model (11), and they ensure that the long-time 
large-scale dynamics (11) would be the desired hydrodynamic equations. The problem is then reduced to finding a parametric expression for the equilibrium population such that all the constraints are satisfied.

However, in order to construct minimal entropic model we take a different root. To begin with, we remind the reader of the important observation [13] on the relation between the discretization of the velocity space and the well known Grad's moment method [14]. Namely, if discrete velocities are constructed from zeros of the Hermite polynomials, the method of discrete velocity is essentially equivalent to Grad's moment method based on the expansion of the distribution function around a fixed Maxwellian distribution function. The natural extension of this approach towards entropic schemes is to link the discrete velocity model not to the Grad's method but instead to the entropic Grad's method (the maximum entropy approximation) [15. To this end, Boltzmann's $H$ function, $H=\int F \ln F d \mathbf{c}$, where $F(\mathbf{x}, \mathbf{c})$ is the one-particle distribution function, $\mathbf{x}$ is the position vector, and $\mathbf{c}$ is the continuous velocity, is evaluated using the Gauss-Hermite quadrature. This gives the discrete form of the $H$ function,

$$
H_{\left\{w_{i}, \mathbf{c}_{i}\right\}}=\sum_{i=1}^{b} f_{i} \ln \left(\frac{f_{i}}{w_{i}}\right) .
$$

Here $w_{i}$ is the weight associated with the $i$ th discrete velocity $\mathbf{c}_{i}$, and the particles mass and the Boltzmann constant $k_{\mathrm{B}}$ are set equal to the unity. Discrete velocity populations $f_{i}(\mathbf{x})$ are related to values of the distribution function at the nodes of the quadrature as $f_{i}(\mathbf{x})=w_{i}\left(2 \pi T_{0}\right)^{(D / 2)} \exp \left(c_{i}^{2} /\left(2 T_{0}\right)\right) F\left(\mathbf{x}, \mathbf{c}_{i}\right)$. Note that the weights are incorporated into the definition of $f_{i}$, and that the Maxwell velocity distribution function has been factored out because this Gaussian probability distribution is taken into account through the GaussHermite quadrature. Discrete-velocity entropy functions (3) for various $\left\{w_{i}, \mathbf{c}_{i}\right\}$ is the unique input for all our constructions below.

We shall first consider the isothermal hydrodynamics. It is known 13 that the minimal set of discrete velocities needed to reconstruct Navier-Stokes equations corresponds to zeroes of the third order Hermite polynomials in $c_{i} / \sqrt{2 T_{0}}$. For $D=1$, the three discrete velocities are $c=\left\{-\sqrt{3 T_{0}}, 0, \sqrt{3 T_{0}}\right\}$, whereas the corresponding weights are $w=\left\{\frac{1}{6}, \frac{2}{3}, \frac{1}{6}\right\}$ respectively. In higher dimensions, the discrete velocities are tensor products of the discrete velocities in one dimension and the weights are constructed by multiplying weights associ- 
ated with each component direction. In this way we construct the entropy function (3). It is important to remark that for $D=2$ the entropy function thus obtained coincides with the one derived in Ref. [10] by a completely different kind of argument.

The discrete-velocity local equilibrium is the minimizer of the corresponding entropy function under the fixed density and the momentum (2). The explicit solution to this conditional minimization problem in $D$ dimensions reads:

$$
f_{i}^{\mathrm{eq}}=\rho w_{i} \prod_{\alpha=1}^{D}\left(2-\sqrt{1+u_{\alpha}^{\prime 2}}\right)\left(\frac{\frac{2}{\sqrt{3}} u_{\alpha}^{\prime}+\sqrt{1+u_{\alpha}^{\prime 2}}}{1-u_{\alpha}^{\prime} /(\sqrt{3})}\right)^{c_{i \alpha} / \sqrt{3} c_{\mathrm{s}}}
$$

where $c_{\mathrm{s}}=\sqrt{T_{0}}$ is the speed of the sound and the dimensionless velocity $u_{\alpha}^{\prime}=\left(u_{\alpha} / c_{\mathrm{s}}\right)$. Note that the exponent, $\left(c_{i \alpha} /\left(\sqrt{3} c_{\mathrm{s}}\right)\right)$, in Eq. (4) takes the values \pm 1 , and 0 only and the resulting expressions for the equilibrium can be simplified in each dimensions. Equilibria (四) are positive definite for $u_{\alpha}<\sqrt{3} c_{\mathrm{s}}$. Without going into details of derivation, we mention that the equilibrium (4) is the product of $D$ one-dimensional solutions, see for example Ref. [7]. This factorization is pertinent to the derivation, and is quite similar to the familiar property of Maxwell's distribution function.

We stress that none of the conditions for the higher-order equilibrium moments have been used while deriving (4). Relevant higher-order moments of the equilibrium distribution, needed to establish isothermal hydrodynamics in the framework of the ChapmanEnskog method are the equilibrium pressure tensor, $P_{\alpha \beta}^{\mathrm{eq}}=\sum_{i} f_{i}^{\mathrm{eq}} c_{i \alpha} c_{i \beta}$, and the equilibrium third-order moments, $Q_{\alpha \beta \gamma}^{\mathrm{eq}}=\sum_{i} f_{i}^{\mathrm{eq}} c_{i \alpha} c_{i \beta} c_{i \gamma}$. A direct computation shows that the present equilibrium results in the correct pressure tensor (correct means as obtained in the continuous kinetic theory) to the order $O\left(u^{4}\right)$, which is sufficient to simulations of the Navier-Stokes dynamics at small Mach number. For example, for $D=2$, even at high dimensionless velocity, $u_{\alpha}=0.25 \sqrt{T_{0}}$, the error in the pressure is less than $2 \%$. Moreover, the third-order moment is even more accurate as compared to the standard quadratic polynomial equilibrium, which leads to a $O\left(u^{3}\right)$ error in simulations [16]. In the present case, only the diagonal component $Q_{\alpha \alpha \alpha}^{\mathrm{eq}}$ have the same linear accuracy as in the standard LBM, whereas all the $D^{3}-D$ non-diagonal components of $Q_{\alpha \beta \gamma}^{\mathrm{eq}}$ are correct up to order $O\left(u^{5}\right)$.

The set of the discrete velocities used in the present case is the same as standard D2Q9 and D3Q27 model of the lattice Boltzmann method. The expansion of the equilibrium to the order $O\left(u^{2}\right)$ coincides with the polynomial equilibria used in the lattice Boltzmann method. 
Thus, it is not surprising that D2Q9 model is more stable than any other LBM. However, due to the enhanced numerical stability and accuracy in the heat flux, the use of the positive definite equilibrium (Eq. (4) is more preferable in comparison to its expanded form in the isothermal lattice BGK method.

In precisely the same way, the minimal entropic kinetic model for the nonisothermal case requires zeroes of fourth-order Hermite polynomials. For $D=1$, the four discrete velocities and corresponding weights of Gauss-Hermite quadrature are $c=( \pm a, \pm b)$, and $w=\left(w_{a}, w_{b}\right)=\left(T_{0} /\left(4 a^{2}\right), T_{0} /\left(4 b^{2}\right)\right)$, respectively, where $a=\sqrt{3-\sqrt{6}}\left(T_{0}\right)^{1 / 2}$, and $b=$ $\sqrt{3+\sqrt{6}}\left(T_{0}\right)^{1 / 2}$. The minimizer of the $H$ function (3) corresponding to the velocity set and weights just described, and subject to the constraints (2), may be written as

$$
f_{i}^{\mathrm{eq}}= \begin{cases}\frac{\rho\left(b^{2}-T-u^{2}\right)}{b^{2}-a^{2}} \frac{\exp \left(B c_{i}\right)}{\exp (B a)+\exp (-B a)} & \text { if } c_{i}= \pm a, \\ \frac{\rho\left(u^{2}+T-a^{2}\right)}{b^{2}-a^{2}} \frac{\exp \left(B c_{i}\right)}{\exp (B b)+\exp (-B b)} & \text { if } c_{i}= \pm b\end{cases}
$$

Lagrange multiplier $B$, corresponding to the momentum constraint, has the following series representation:

$$
B=\frac{u}{E}-\frac{u^{3}}{3}\left[\frac{a^{2} b^{2}}{E^{4}}-\frac{\left(a^{2}+b^{2}\right)}{E^{3}}\right]+O\left(u^{5} E^{-5}\right),
$$

where $E=u^{2}+T$ is the total energy density [Notice that Eq. (6) is not an expansion in powers of velocity $u$, rather, in terms of $u^{n} E^{-m}$.] Equilibrium distribution (5) exists within a positivity interval, $a^{2} \leq T+u^{2} \leq b^{2}$.

In higher dimensions, the set of discrete velocities is formed by taking tensor product of the discrete velocities in $D=1$, and the weights are products of corresponding quantities. In order to evaluate Lagrange multipliers in the formal solution to the minimization problem, $f_{i}^{\text {eq }}=w_{i} \exp \left(A+\boldsymbol{B} \cdot \mathbf{c}_{i}+C c_{i}^{2}\right)$, we first make an important observation that they can be computed exactly for $\mathbf{u}=0$ and any temperature $T$ within the positivity interval, $a^{2}<T<$ $b^{2}:$

$$
\begin{array}{r}
B_{\alpha}=0, \quad C_{0}=\frac{1}{\left(b^{2}-a^{2}\right)} \log \left(\frac{w_{a}\left(T-a^{2}\right)}{w_{b}\left(b^{2}-T\right)}\right), \\
A_{0}=\log \left(\frac{\rho\left(b^{2}-T\right)^{D}}{\left(2 w_{a}\right)^{D}\left(b^{2}-a^{2}\right)^{D}}\right)-D a^{2} C_{0} .
\end{array}
$$

With this, we find the equilibrium at zero average velocity and arbitrary temperature, 


$$
\begin{aligned}
f_{i}^{\mathrm{eq}}= & \frac{\rho w_{i}}{2^{D}\left(b^{2}-a^{2}\right)^{D}} \times \\
& \prod_{\alpha=1}^{D}\left(\frac{b^{2}-T}{w_{a}}\right)^{\left(\frac{b^{2}-c_{i \alpha}^{2}}{b^{2}-a^{2}}\right)}\left(\frac{T-a^{2}}{w_{b}}\right)^{\left(\frac{c_{i \alpha}^{2}-a^{2}}{b^{2}-a^{2}}\right)} .
\end{aligned}
$$

Factorization over spatial components is clearly seen in this solution. Once the exact solution for zero velocity is known, extension to $\mathbf{u} \neq 0$ is easily found by perturbation. The first few terms of this expansion are:

$$
\begin{aligned}
A & =A_{0}-\frac{T}{\left(T-a^{2}\right)\left(b^{2}-T\right)} u^{2}+O\left(u^{4}\right), \\
B_{\alpha} & =\frac{u_{\alpha}}{T}+\frac{\left(T-T_{0}\right)^{2}}{2 D T^{4}}\left(D u_{\beta} u_{\theta} u_{\gamma} \delta_{\alpha \beta \gamma \theta}-3 u^{2} u_{\alpha}\right)+O\left(u^{5}\right), \\
C & =C_{0}+\frac{a^{2}\left(b^{2}-T\right)-T\left(b^{2}-3 T\right)}{2 D T^{2}\left(T-a^{2}\right)\left(b^{2}-T\right)} u^{2}+O\left(u^{4}\right) .
\end{aligned}
$$

For the actual numerical implementation, the equilibrium distribution function can be calculated analytically, up to any order of accuracy required, by this procedure. Note that dependence on the temperature in the above equations is a nonperturbative result.

In order to establish the hydrodynamic equations corresponding to the present model, apart from the equilibrium pressure tensor $P_{\alpha \beta}^{\mathrm{eq}}$ and the equilibrium third moment $Q_{\alpha \beta \gamma}^{\mathrm{eq}}$, one needs to check also the fourth order moment, $R_{\alpha \beta}^{\mathrm{eq}}=\sum_{i} c_{i \alpha} c_{i \beta} c^{2} f_{i}^{\mathrm{eq}}$. A direct computation shows that the equilibrium stress tensor is exact from the computational standpoint (accurate at least up to the order $O\left(u^{8}\right)$ ). The third moments $Q_{\alpha \beta \gamma}$ are accurate up to the order $O\left(u \theta^{2}\right), O\left(u^{3} \theta\right)$ and $O\left(u^{5}\right)$, where $\theta=\left(T_{0}-T\right) / T_{0}$ is the deviation of the temperature from the reference value. Finally, the fourth moments, $R_{\alpha \beta}$ are accurate to the order $O\left(\theta^{2}\right), O\left(u^{2} \theta^{2}\right), O\left(u^{4}\right)$. Thus the Navier-Stokes-Fourier dynamics is recovered to the order $O\left(u^{3}, \theta^{2}\right)$. Notice that if the temperature is fixed at the reference value $T=T_{0}$, the moment $Q_{\alpha \beta \gamma}^{\mathrm{eq}}$ becomes exact to the order $O\left(u^{5}\right)$, unlike in the second-order accurate standard lattice Boltzmann models and the isothermal model constructed above.

The local equilibrium entropy, $S=-k_{\mathrm{B}} H_{\left\{w_{i}, \mathbf{c}_{i}\right\}}\left(f^{\mathrm{eq}}\right)$, for the nonisothermal model satisfies the usual expression for the entropy of the ideal monoatomic gas to the overall order of approximation of the method, $S=\rho k_{\mathrm{B}} \ln \left(T^{D / 2} / \rho\right)+O\left(u^{4}, \theta^{2}\right)$. Thus, the present model is able to retain the thermodynamics up to the accuracy of the method. To the best of our knowledge, this is the first discrete velocity model which is fully consistent with thermodynamics. 
When the single relaxation time BGK model (1) is used with the present nonisothermal equilibrium, the resulting transport coefficients are as follows: For $D=1$, the kinematic viscosity $\nu$ is equal to zero, while the thermal conductivity $\kappa$ is $\kappa=(3 / 2)(\tau \rho) T$. For $D>1$, we have $\nu=(\tau \rho) T$, and $\kappa=((D+2) / 2)(\tau \rho) T$ [we recall that one needs to renormalize the relaxation time in the BGK model as $\tau^{\prime}=\tau \rho$, in order to obtain density-independent transport coefficients].

Finally, we present here some details of the discretization scheme for model kinetic Eq. (1). In a kinetic model there are two time scales one associated with convection and the other one with collisions, where the timescale of collisions is much smaller than that of convection. In order to have an efficient simulation scheme for the hydrodynamics, it is desirable to follow the time scale of the convection in the simulation. To achieve such a scheme we propose to simulate discrete kinetic equation

$$
f_{i}(\mathbf{x}, \delta t)=L_{\left\{\mathbf{c}_{i}, \delta t\right\}} \cdot\left[f_{i}(\mathbf{x}, 0)+\frac{\alpha}{\left(2 \tau^{\prime}+\delta t\right)}\left(f_{i}^{\mathrm{eq}}(\mathbf{x}, 0)-f_{i}(\mathbf{x}, 0)\right)\right],
$$

were $L_{\left\{\mathbf{c}_{i}, \delta t\right\}}$ is a linear convection operator and is defined by the relation $L_{\left\{\mathbf{c}_{i}, \delta t\right\}} \cdot g(\mathbf{x}, t)=$ $g\left(\mathbf{x}-\mathbf{c}_{i} \delta t, t\right)$, and the parameter $\alpha$ is defined by the condition:

$$
H_{\left\{w_{i}, \mathbf{c}_{i}\right\}}\left(f_{i}(\mathbf{x}, 0)\right)=H_{\left\{w_{i}, \mathbf{c}_{i}\right\}}\left(f_{i}(\mathbf{x}, 0)+\alpha\left(f_{i}^{\mathrm{eq}}(\mathbf{x}, 0)-f_{i}(\mathbf{x}, 0)\right)\right) .
$$

Close to the local equilibrium the parameter $\alpha$ is equals to $2 \delta t$ [8]. The details of the implementation of Eq. (10) are presented in the Ref. [8]. The essence of the Eq. (9) and $(\sqrt{10})$ is to separate the convection, which just shifts the population in space (operator $\left.L_{\left\{\mathbf{c}_{i}, \delta t\right\}}\right)$, from the collisions which represent the relaxation of the populations towards the equilibrium. After each convection step, the length of collision step is restricted by the condition of the entropy conservation during the collision (Eq.10). This lumping of many short collision steps ensures that rapid convergence towards hydrodynamics is achieved. The details of the derivation of Eq. (9) and Eq. (10) from the model kinetic equation (四) will be presented elsewhere.

In the isothermal case, this model can be implemented in a efficient manner by taking the uniform grid spacing in each direction as $\delta x$ and the time step as $\delta t=\delta x / \sqrt{3 T_{0}}$. This means $\mathbf{x}-\mathbf{c}_{i} \delta t$ is always a grid point and the resulting method is LBM. In the nonisothermal case, the error in the approximations can be minimized by choosing the timestep as $\delta t=$ $3 \delta x / b$ and performing a dimensional splitting, for example in the two dimensional case $L_{\left\{c_{i x}, c_{i y}, \delta t\right\}} \cdot g(\mathbf{x}, t)=L_{\left\{c_{i x}, 0, \delta t\right\}} \cdot\left[L_{\left\{0, c_{i y}, \delta t\right\}} \cdot g(\mathbf{x}, t)\right]$. 
For directions in which velocity component equals $a$, we propose to discretize the one dimensional convection operator by the Beam-Warming operator [17] defined as $L_{\{a, 3 \delta x / b\}}$. $g(x, t)=0.02432 g(x, t)+0.99784 g(x-\delta x, t)+0.02216 g(x-2 \delta x, t)$. We remind the reader that as the CFL number approaches the value of unity (in the present case equals $3 a / b=0.95351$ ), the error of the discretization vanishes [17]. The present discretization scheme, in contrast to the earlier proposed finite-volume and finite-difference methods [1] for solving Eq. (11), is using a large time step (of the order $O(\delta x)$ ) like LBM.

In order to check the effectiveness of the algorithm for the nonisothermal case, we have performed the simulation of the Taylor vortex flow. The flow is in the isothermal and the incompressible set up, which is achieved in the simulation through the initial condition. This problem is chosen to validate the theoretical expression for the viscosity and the discretization procedure. The flow is completely characterized by the analytical solution $u(x, y, t)=\nabla \times\left[\left(u_{0} / k_{2}\right) \exp \left[-\nu\left(k_{1}^{2}+k_{2}^{2}\right) t\right] \cos \left(k_{1} x\right) \cos \left(k_{2} y\right)\right]$. In the simulation, we have chosen $u_{0}=0.0001, k_{1}=1, k_{2}=4$. The result in Fig.11 shows that the discretization procedure is working well even at very short times.

To conclude, in this Letter we have derived minimal entropic kinetic models of the Boltzmann equation for both isothermal and nonisothermal hydrodynamic simulations. The resulting models have correct hydrodynamics, they are equipped with the appropriate $H$ function, and also they are thermodynamically consistent. A simple discretization scheme is proposed for the simulation. In the isothermal case, we have found analytically the corresponding local equilibrium in closed form (4), and thus proposed a isothermal LBM with correct $H$ theorem.

* Corresponding author. E-mail: ikarlin@ifp.mat.ethz.ch

[1] S. Succi, The Lattice Boltzmann Equation for Fluid Dynamics and Beyond (Oxford University Press, Oxford, 2001).

[2] V. M. Kendon, J.-C. Desplat, P. Bladon, and M. E. Cates, Phys. Rev. Lett. 83, 576 (1999).

[3] S. Chen and G. D. Doolen, Annu. Rev. Fluid Mech. 30, 329 (1998).

[4] W. Miller, S. Succi, and D. Mansutti, Phys. Rev. Lett. 70, 1339 (1993).

[5] S. Succi, I. V. Karlin, and H. Chen, Rev. Mod. Phys., to appear 74 (2002). 
[6] G. R. McNamara, A. L. Garcia, and B. J. Alder, J. Stat. Phys. 81, 395 (1995).

[7] S. Ansumali and I. V. Karlin, Phys. Rev. E 62, 7999 (2000).

[8] S. Ansumali and I. V. Karlin, J. Stat. Phys. 107, 291 (2002).

[9] S. Ansumali and I. V. Karlin, Phys. Rev. E 65, 056312 (2002).

[10] I. V. Karlin, A. Ferrante, and H. C. Öttinger, Europhys. Lett. 47, 182 (1999).

[11] C. Cercignani, Transp. Theory Stat. Phys. 23, 1 (1994).

[12] C. Cercignani, Theory and Application of the Boltzmann Equation (Scottish Academic Press, Edinburgh, 1975).

[13] X. Shan and X. He, Phys. Rev. Lett. 80, 65 (1998).

[14] H. Grad, Handbuch der Physik XII (1958).

[15] A. M. Kogan, J. Appl. Math. Mech. 29, 130 (1965).

[16] Y. H. Qian and S. A. Orszag, Europhys. Lett. 21, 255 (1993).

[17] C. B. Laney, Computational gas dynamics (Cambridge University Press,Cambridge, 1998).

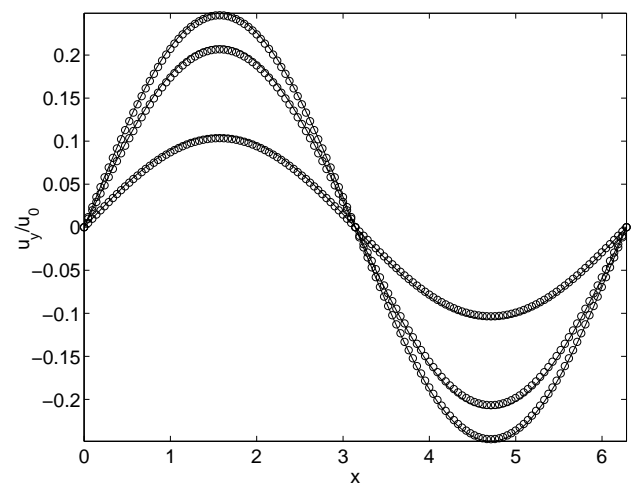

FIG. 1: Simulation of the two dimension Taylor vortex flow. The velocity profiles at $y=\pi$ at three different times $t=0.03,10,50$ are shown. The solid lines represent analytical results with viscosity $\nu=\tau^{\prime} T$, circles represent simulation results. All the quantities are given in dimensionless units. 